\title{
Cosmic Strings Stabilized by Fermion Fluctuations
}

\author{
Herbert Weigel \\ Physics Department, Stellenbosch University, \\ Matieland 7602, South Africa \\ weigel@sun.ac.za \\ Markus Quandt \\ Institute for Theoretical Physics, Tübingen University \\ D-72076 Tübingen, Germany \\ quandt@tphys.physik.uni-tuebingen.de \\ Noah Graham \\ Department of Physics, Middlebury College \\ Middlebury, VT 05753, USA \\ ngraham@middlebury.edu \\ Received Day Month Year \\ Revised Day Month Year
}

\begin{abstract}
We provide a thorough exposition of recent results on the quantum stabilization of cosmic strings. Stabilization occurs through the coupling to a heavy fermion doublet in a reduced version of the standard model. The study combines the vacuum polarization energy of fermion zero-point fluctuations and the binding energy of occupied energy levels, which are of the same order in a semi-classical expansion. Populating these bound states assigns a charge to the string. Strings carrying fermion charge become stable if the Higgs and gauge fields are coupled to a fermion that is less than twice as heavy as the top quark. The vacuum remains stable in the model, because neutral strings are not energetically favored. These findings suggest that extraordinarily large fermion masses or unrealistic couplings are not required to bind a cosmic string in the standard model.
\end{abstract}

Keywords: Cosmic Strings, Vacuum Polarization Energy, Spectral Method, Fermion Fluctuations

PACS numbers: 11.27.+d, 03.65.Ge, 14.65.Jk

\section{Introduction}

It is well-known that the electroweak standard model and many of its extensions have the potential to support string-like configurations that are the particle physics analogs of vortices or magnetic flux tubes in condensed matter physics. Such objects are usually called cosmic strings to distinguish them from the fundamental variables in string theory, and also to indicate that they typically stretch over cosmic length scales. They are also called electroweak strings or $Z_{\text {-strings }} 1-3$ because the $Z_{-}$ 
component of the electroweak gauge boson acquires the structure of the Abelian Nielsen-Olesen vortex ${ }^{4}$.

Such strings may have emerged copiously in the early universe at interfaces between regions of different vacuum expectation values of the Higgs field(s) in several stages after the Big Bang until electroweak symmetry breaking was reached. If strings are absolutely stable they should have survived and we should be able to observe them today. In the electroweak standard model topologically stable strings are ruled out, but the absence of this particular stabilizing mechanism does not imply that electroweak strings are unstable or irrelevant for particle physics. While their direct gravitational effects are negligible, $Z$-strings can still be relevant for cosmology at a sub-dominant leve $\sqrt{516}$. Their most interesting consequences originate, however, from their coupling to the standard model fields. $Z$-strings provide a source for primordial magnetic fields 3 and they also offer a scenario for baryogenesis with a second order phase transition $7 / 8$. In contrast, a strong first order transition as required by the usual bubble nucleation scenario is unlikely in the electroweak standard mode $[9]$ without non-standard additions such as supersymmetry or higherdimensional operators 10 .

These interesting effects are only viable if $Z$-strings are energetically stabilized by their coupling to the remaining quantum fields. The most important contributions are expected to come from (heavy) fermions, since their quantum energy dominates in the limit $N_{C} \rightarrow \infty$, where $N_{C}$ is the number of QCD colors or of any other internal degree of freedom. The Dirac spectrum in typical string backgrounds is deformed to contain either an exact or near zero mode, so that fermions can substantially lower their energy by binding to the string. This binding effect can overcome the classical energy required to form the string background. However, the remaining spectrum of modes is also deformed and for consistency its contribution (the vacuum polarization energy) must be taken into account as well. Heavier fermions are expected to provide more binding since the energy gain per fermion charge is higher; a similar conclusion can also be obtained from decoupling arguments 11 .

A number of previous studies have investigated quantum properties of string configurations. Naculich 12 has shown that in the limit of weak coupling, fermion fluctuations destabilize the string. The quantum properties of $Z$-strings have also been connected to non-perturbative anomalies 13 . The emergence or absence of exact neutrino zero modes in a $Z$-string background and the possible consequences for the string topology were investigated in Ref. 14. A first attempt at a full calculation of the fermionic quantum corrections to the $Z$-string energy was carried out in Ref. 15. Those authors were only able to compare the energies of two string configurations, rather than comparing a single string configuration to the vacuum because of limitations arising from the non-trivial behavior at spatial infinity. We will discuss a solution to this obstacle in detail below. The fermionic vacuum polarization energy of the Abelian Nielsen-Olesen vortex has been estimated in Ref. 16 with regularization limited to the subtraction of the divergences in the heat-kernel 
expansion. Quantum energies of bosonic fluctuations in string backgrounds were calculated in Ref. 17. Finally, the dynamical fields coupled to the string can also result in (Abelian or non-Abelian) currents running along the core of the string. The time evolution of such structured strings was studied in Ref. 18, where the current was induced by the coupling to an extra scalar field.

This presentation is based on a number of publications $19-22$ regarding the contribution of fermion quantum corrections to the vacuum polarization energy of a straight and infinitely long cosmic string that appeared over the last year or so. Technical details underlying the results given here can be learned from those publications, $c f$. in particular the appendices of Ref. 22 .

\section{Model}

For the current investigation the fermion doublet will be considered degenerate so that the introduction of a matrix notation for the Higgs field is appropriate. Then the string configuration reads

$$
\Phi=v f_{H}(\rho)\left(\begin{array}{cc}
\sin \left(\xi_{1}\right) \mathrm{e}^{-i n \varphi} & -i \cos \left(\xi_{1}\right) \\
-i \cos \left(\xi_{1}\right) & \sin \left(\xi_{1}\right) \mathrm{e}^{i n \varphi}
\end{array}\right)
$$

for the Higgs field and

$$
\vec{W}=n \sin \left(\xi_{1}\right) \frac{f_{G}(\rho)}{g \rho} \hat{\varphi}\left(\begin{array}{cc}
\sin \left(\xi_{1}\right) & i \cos \left(\xi_{1}\right) \mathrm{e}^{-i n \varphi} \\
-i \cos \left(\xi_{1}\right) \mathrm{e}^{i n \varphi} & -\sin \left(\xi_{1}\right)
\end{array}\right)
$$

for the gauge boson (in temporal gauge). The variables $\rho$ and $\varphi$ are polar coordinates in the plane perpendicular to the string, while the Higgs vacuum expectation value $v$ and the gauge coupling constant $g$ are model parameters. The string configuration involves profile functions $f_{H}$ and $f_{G}$ which are the analogs of the Nielsen-Olesen vortex profiles with boundary conditions

$$
\begin{array}{ll}
\rho \longrightarrow 0: & f_{G}, f_{H} \longrightarrow 0 \\
\rho \longrightarrow \infty: & f_{G}, f_{H} \longrightarrow 1 .
\end{array}
$$

The integer $n$ is the winding of the string, for which we will typically take $n=1$ in numerical calculations. Finally, the (variational) parameter $\xi_{1}$ measures the relative strength of the Higgs and gauge boson components of the string.

We will consider a modified version of the electroweak standard model which only has a $S U(2)_{L}$ gauge symmetry. This modification is equivalent to a vanishing Weinberg angle. Then the (classical) boson part of the Lagrangian reads

$$
\mathcal{L}_{\text {bos }}=-\frac{1}{2} \operatorname{tr}\left(G^{\mu \nu} G_{\mu \nu}\right)+\frac{1}{2} \operatorname{tr}\left(D^{\mu} \Phi\right)^{\dagger} D_{\mu} \Phi-\frac{\lambda}{2} \operatorname{tr}\left(\Phi^{\dagger} \Phi-v^{2}\right)^{2},
$$

with the covariant derivative $D_{\mu}=\partial_{\mu}-i g W_{\mu}$ and the $S U(2)$ field strength tensor

$$
G_{\mu \nu}=\partial_{\mu} W_{\nu}-\partial_{\nu} W_{\mu}-i g\left[W_{\mu}, W_{\nu}\right] .
$$


The boson masses are determined from $g$ and $v$ and the Higgs self-coupling $\lambda$ as $m_{\mathrm{W}}=g v / \sqrt{2}$ and $m_{\mathrm{H}}=2 v \sqrt{\lambda}$ for the gauge and Higgs bosons, respectively. The interaction with the fermion doublet is described by the Lagrangian

$$
\mathcal{L}_{\mathrm{fer}}=i \bar{\Psi}\left(P_{L} \not D+P_{R} \not\right) \Psi-f \bar{\Psi}\left(\Phi P_{R}+\Phi^{\dagger} P_{L}\right) \Psi \quad \text { with } \quad P_{R, L}=\frac{1}{2}\left(\mathbb{1} \pm \gamma_{5}\right) .
$$

Upon spontaneous symmetry breaking the Yukawa coupling $f$ induces a fermion mass $m=v f$. The similarity with the standard model of particle physics suggests the model parameters

$$
g=0.72, \quad v=177 \mathrm{GeV}, \quad m_{\mathrm{H}}=140 \mathrm{GeV}, \quad f=0.99
$$

where we have taken the fermion doublet to have the mass of the top quark. In our numerical search for a stable string, we will also study other model parameters, $c f$. section 5.

The classical energy per unit length of the string is solely governed by $\mathcal{L}_{\text {bos }}$ :

$$
\begin{gathered}
\frac{E_{\mathrm{cl}}}{m^{2}}=2 \pi \int_{0}^{\infty} \rho d \rho\left\{n^{2} \sin ^{2} \xi_{1}\left[\frac{2}{g^{2}}\left(\frac{f_{G}^{\prime}}{\rho}\right)^{2}+\frac{f_{H}^{2}}{f^{2} \rho^{2}}\left(1-f_{G}\right)^{2}\right]\right. \\
\left.+\frac{f_{H}^{\prime 2}}{f^{2}}+\frac{\mu_{H}^{2}}{4 f^{2}}\left(1-f_{H}^{2}\right)^{2}\right\},
\end{gathered}
$$

where the dimensionless radial integration variable is related to the physical radius by $\rho_{\text {phys }}=\rho / m$ and $\mu_{H} \equiv m_{\mathrm{H}} / m$.

The central object of the present investigation is the fermion contribution to the energy. It will obtained from the solutions to the Dirac equationa in the twodimensional plane perpendicular to the string

$$
H \Psi_{n}=\omega_{n} \Psi_{n} \quad \text { with } \quad H=-i\left(\begin{array}{cc}
0 & \vec{\sigma} \cdot \hat{\rho} \\
\vec{\sigma} \cdot \hat{\rho} & 0
\end{array}\right) \partial_{\rho}-\frac{i}{\rho}\left(\begin{array}{cc}
0 & \vec{\sigma} \cdot \hat{\varphi} \\
\vec{\sigma} \cdot \hat{\varphi} & 0
\end{array}\right) \partial_{\varphi}+H_{\mathrm{int}}
$$

where the single particle Hamiltonian, $H$ is extracted from $\mathcal{L}_{\text {fer }}$. The interaction part, $H_{\text {int }}$ depends on the chosen gauge. A specific choice of gauge, which accommodates the subtleties at large $\rho$, will be discussed in Sec. 3.3. The spectrum of the Dirac operator consists of bound state solutions with discrete eigenvalues $\omega_{n}=\epsilon_{j}$ and continuous scattering solutions whose eigenvalues $\omega$ are labeled by momentum $k$, i.e. $\omega=\sqrt{k^{2}+m^{2}}$. Finally, the Dirac Hamiltonian for the string background anti-commutes with $\alpha_{3}=\gamma^{0} \gamma^{3}$. Thus the spectrum is charge conjugation invariant and it suffices to consider the non-negative eigenvalues.

${ }^{\mathrm{a}}$ We use the standard representation with $\gamma_{0}=\operatorname{diag}(1,1,-1,-1)$. 


\section{Formalism}

The vacuum polarization energy is the renormalized sum of the changes of the zero-point energies of fermions in the background of a static configuration

$$
E_{\mathrm{vac}}=-\left.\frac{\hbar}{2} \sum_{n}\left(\omega_{n}-\omega_{n}^{(0)}\right)\right|_{\mathrm{ren}}=-\frac{\hbar}{2} \sum_{j} \epsilon_{j}-\hbar \int_{0}^{\infty} d k \omega_{k} \Delta \rho_{\mathrm{ren}}(k) .
$$

Here $\omega_{n}$ are the energy eigenvalues in the presence of the string as obtained from the Dirac equation (9), and $\omega_{n}^{(0)}$ are their free counterparts. The overall sign has been chosen to describe fermionic vacuum fluctuations; for bosons it must be altered. In the second part of equation (10) the changes of the single particle energies are rewritten as the contribution from distinct bound states $\left(\epsilon_{j}\right)$ and the (renormalized) change of the density of scattering states, $\Delta \rho_{\text {ren }}(k)$. These states obey the standard dispersion relation $\omega_{k}=\sqrt{k^{2}+m^{2}}$ with the mass $m$ of the quantum fluctuations. In the above, the factor $\hbar$ has been made explicit to stress that $E_{\mathrm{vac}}$ is a quantum effect. As in the previous section natural units $(\hbar=1$ and $c=1)$ will be adopted in the remainder of this paper.

The computation of the fermion contribution to the vacuum polarization energy of a cosmic string proceeds in three stages. First, spectral methods are employed to express this energy in form of scattering data 23 . The equivalence of terms in the Born series and Feynman diagrams makes it possible to impose standard renormalization conditions. In a second step this approach is extended to accommodate configurations that are translationally invariant in one or more spatial directions, using the so-called interface formalism 24 . Third, we have to cope with the fact that the cosmic string configuration has a non-trivial structure at spatial infinity. Because the fields approach a pure gauge rather than zero, the direct application of spectral methods is impossible. Rather a particular subset of gauges has to be adopted to define a well-behaved scattering problem 20 .

\subsection{Spectral Methods}

The basic idea of the spectral approach is to express $\Delta \rho_{\text {ren }}(k)$ as the momentum derivative of the phase shifts of the scattering states 25 . Then

$$
E_{\mathrm{vac}}=-\frac{1}{2} \sum_{j} \epsilon_{j}-\sum_{\ell} D_{\ell} \int_{0}^{\infty} \frac{d k}{2 \pi} \omega_{k} \frac{d}{d k}\left[\delta_{\ell}(k)\right]_{\mathrm{ren}} .
$$

It is implicitly assumed that the system allows for a partial wave decomposition with degeneracy factor $D_{\ell}$ for the partial wave of (generalized) angular momentum $\ell$. The major concern in eq. (11) is renormalization. As it stands, in three spatial dimensions the vacuum polarization energy, eq. (11), is quadratically divergent in the ultra-violet. For large momenta the Born series adequately represents the phase 
shift $\mathrm{b}$. We therefore consider

$$
E^{(N)}=-\frac{1}{2} \sum_{j} \epsilon_{j}-\sum_{\ell} D_{\ell} \int_{0}^{\infty} \frac{d k}{2 \pi} \omega_{k} \frac{d}{d k}\left[\delta_{\ell}(k)\right]_{N},
$$

where the subscript on the phase shift indicates the subtraction of the $N$ leading terms of the Born series to the phase shift: $\left[\delta_{\ell}\right]_{N}=\delta_{\ell}-\delta_{\ell}^{(1)}-\delta_{\ell}^{(1)}-\ldots-\delta_{\ell}^{(N)}$. Choosing $N$ sufficiently large renders $E^{(N)}$ finite. Technically the Born series is an expansion in powers of $H_{\text {int }}$. The contribution of any such power to the vacuum polarization energy can be associated with a Feynman diagram

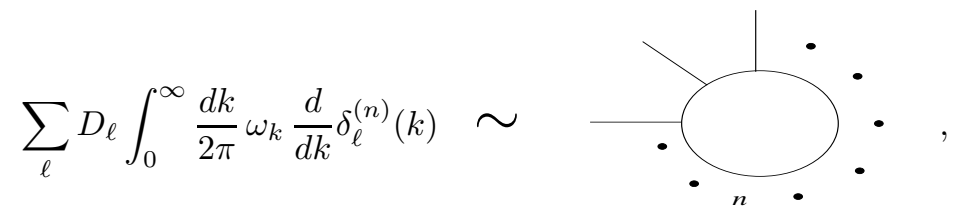

where the loop corresponds to the quantum fluctuation and the external lines represent insertions of the background field, i.e. the interaction of the cosmic string with the fermions. Therefore, the artificial subtraction in equation (12) is the sum over all Feynman diagrams with up to $N$ insertions of the background interaction: $E_{\mathrm{FD}}^{(N)}$. These diagrams can be computed with standard techniques, in particular dimensional regularization can be implemented to handle the ultra-violet divergences.

The most important advantage of shuffling the divergences into Feynman diagrams is, however, that they can be straightforwardly combined with the counterterm contribution to the energy, $E_{\mathrm{CT}}$. The latter is found by substituting the background configuration into the counterterm Lagrangian in just the same way as the classical energy, eq. (8) is obtained from $\mathcal{L}_{\text {bos }}$. In any (multiplicatively) renormalizable theory the counterterm Lagrangian has the same structure as the classical one and a suitable choice of its coupling constants cancels all ultra-violet divergences completely. Then $E_{\mathrm{FD}}^{(N)}+E_{\mathrm{CT}}$ is free of any divergences. The finite pieces of the counterterm coupling constants will be uniquely determined from appropriate conditions describing properties of the particles that are associated with the fields. This procedure will be briefly discussed in section. 4.3. In total, the sum

$$
E_{\mathrm{vac}}=E^{(N)}+E_{\mathrm{FD}}^{(N)}+E_{\mathrm{CT}}
$$

gives an unambiguous result for the vacuum polarization energy once the renormalization conditions are fixed. A first principle derivation of this result based on a quantum field theoretic formulation of the energy momentum momentum tensor rather and the analytic properties of the Greens function for scattering boundary conditions is presented in Ref. 26 .

${ }^{\mathrm{b}}$ For small momenta it does generally not converge towards the exact phase shift, in particular when bound states are present. 


\subsection{Interface Formalism}

Equation (14) is perfectly suited for a computation of the vacuum polarization energy for static configurations that allow for a full decomposition into partial waves. However, this is not the case for the cosmic string which is translationally invariant along the $\hat{z}$-axis in coordinate space. In this scenario the wave-function of the quantum fluctuation factorizes into

$$
\Psi(\vec{x}, t) \sim \mathrm{e}^{-i \omega t} \mathrm{e}^{i p z} \psi_{k}(\vec{\rho})
$$

so that we have a scattering problem in the plane perpendicular to the string, and the total dispersion relation $\omega=\sqrt{p^{2}+k^{2}+m^{2}}=\sqrt{p^{2}+\omega_{k}^{2}}$. This implies the replacements $\epsilon_{j} \rightarrow \sqrt{p^{2}+\epsilon_{j}^{2}}$ and $\omega_{k} \rightarrow \sqrt{p^{2}+\omega_{k}^{2}}$ in equation (12) so that an integration over $p$ (with measure $d p / 2 \pi$ ) yields the vacuum polarization energy per unit length of the string. In doing so an immediate obstacle arises. The scattering data do not depend on the momentum $p$ along the symmetry axis. Hence for any $N$, this $p$-integral will not be finite. A careful analysis treats the $p$-integral in dimensional regularization 24

$$
\left.E^{(N)} \sim \frac{\Gamma\left(-\frac{1+d}{2}\right)}{2(4 \pi)^{\frac{d+1}{2}}} \sum_{\ell} D_{\ell}\left\{\sum_{j}\left(\epsilon_{j}\right)^{\frac{d+1}{2}}+\int_{0}^{\infty} \frac{d k}{\pi}\left(k^{2}+m^{2}\right)^{\frac{d+1}{2}} \frac{d}{d k}\left[\delta_{\ell}(k)\right]_{N}\right]\right\}
$$

where $d$ is the analytic dimension of the subspace in which the configuration is translationally invariant. The divergence now manifests itself via the singularity of the $\Gamma$-function coefficient as $d \rightarrow 1$. Due to sum rules for scattering data 27 , which represent generalizations of Levinson's theorem, the expression in curly brackets in eq. (16) vanishes as $d \rightarrow 1$. Hence this limit can indeed be taken 24. Finally, the fermion spectrum in the string background is charge conjugation invariant. Thus $E^{(N)}$ is twice its contribution from the non-negative part of the spectrum,

$$
\begin{aligned}
E^{(N)}=\frac{1}{4 \pi} \sum_{\ell} D_{\ell}\left\{\int_{0}^{\infty} \frac{d k}{\pi}\left[\omega_{k}^{2} \ln \left(\frac{\omega_{k}^{2}}{\mu_{r}^{2}}\right)-k^{2}\right] \frac{d}{d k}\left[\delta_{\ell}(k)\right]_{N}\right. \\
\left.+\sum_{j}\left[\epsilon_{j}^{2} \ln \frac{\epsilon_{j}^{2}}{\mu_{r}^{2}}-\epsilon_{j}^{2}+m^{2}\right]\right\} .
\end{aligned}
$$

Here $\mu_{r}$ is an arbitrary renormalization scale that has no effect on $E^{(N)}$ by exactly the same sum rules. The expression, eq. (17), for $E^{(N)}$ replaces the analog in equation (14). Note that the function multiplying the (Born subtracted) phase shift is of higher power in $k$ than its counterpart before integrating over the momentum $p$ conjugate to the coordinate of translational invariance. Hence $N$ must be increased if directions are added in which the configuration is translationally invariant. This, of course, merely reflects the fact that ultra-violet divergences turn more severe in higher dimensions.

We stress that the expressions obtained so far for the vacuum polarization energy strongly rely on the analytic properties of the scattering data. Furthermore, 
in numerical calculations, we mostly consider them for purely imaginary momenta. This has (at least) two advantages: (i) the oscillating phase shifts turn into exponentially decaying (logarithms of the) Jost functions, and (ii) the momentum integral and the sum over angular momenta may be exchanged. While (i) drastically improves numerical stability, (ii) significantly simplifies the treatment of the logarithmic divergences that emerge at third and fourth order of the Born and Feynman expansions. These technical details are discussed at length in Ref. 22 and briefly addressed in section 3.4 .

\subsection{Choice of Gauge}

We have now established a formalism for computing the vacuum polarization energy of background fields in the string geometry. However, we still have the problem that the string does not induce a well-behaved scattering problem because of its non-trivial structure at spatial infinity. Though gauge invariant combinations of the Higgs and gauge bosons are trivial at spatial infinity, the individual terms in the Born and Feynman series are not gauge invariant and therefore ill-defined. This ambiguity appears because the Dirac Hamiltonian that is obtained by straightforward substitution of the field configuration, eqs. (11) and (2) does not turn into the free Dirac Hamiltonian as $\rho \rightarrow \infty$, but instead becomes $H \rightarrow U^{\dagger}(\varphi) H_{\text {free }} U(\varphi)$. This local gauge transformation acts only on the left-handed fermions, $U(\varphi)=P_{L} \exp \left(i \hat{n} \cdot \vec{\tau} \xi_{1}\right)+P_{R}$ with $\hat{n}=(\cos (n \varphi),-\sin (n \varphi), 0)$. Unfortunately, the gauge transformation $H \rightarrow U(\varphi) H U^{\dagger}(\varphi)$ does not solve the problem for all $\rho \in[0, \infty]$ : Although it would generate vanishing interactions at infinity, it also induces a $1 / \rho^{2}$ potential at the center of the string, $\rho \rightarrow 0$. This might still yield well-defined phase shifts, but the conditions underlying the analyticity of the scattering data are certainly violated by this singular behavior. As argued at the end of the previous section, analyticity is central for numerical feasibility of our approach. As a solution, we can define a radially extended gauge transformation

$$
U(\rho, \varphi)=P_{L} \exp (i \hat{n} \cdot \vec{\tau} \xi(\rho))+P_{R} .
$$

This transformation fixes the gauge and in equation (9) it yields the interaction term

$$
\begin{aligned}
H_{\mathrm{int}}=m f_{H} & {\left[\cos (\Delta)\left(\begin{array}{cc}
\mathbb{1} & 0 \\
0 & -\mathbb{1}
\end{array}\right)+i \sin (\Delta)\left(\begin{array}{cc}
0 & \mathbb{1} \\
-\mathbb{1} & 0
\end{array}\right) \vec{n} \cdot \vec{\tau}\right]+\frac{1}{2} \frac{\partial \xi}{\partial \rho}\left(\begin{array}{cc}
-\vec{\sigma} \cdot \hat{\rho} & \vec{\sigma} \cdot \hat{\rho} \\
\vec{\sigma} \cdot \hat{\rho} & -\vec{\sigma} \cdot \hat{\rho}
\end{array}\right) \vec{n} \cdot \vec{\tau} } \\
+ & \frac{n}{2 \rho}\left(\begin{array}{cc}
-\vec{\sigma} \cdot \hat{\varphi} & \vec{\sigma} \cdot \hat{\varphi} \\
\vec{\sigma} \cdot \hat{\varphi} & -\vec{\sigma} \cdot \hat{\varphi}
\end{array}\right)\left[f_{G} \sin (\Delta) I_{G}(\Delta)+\left(f_{G}-1\right) \sin (\xi) I_{G}(-\xi)\right] \cdot \quad \text { (19) }
\end{aligned}
$$

The new gauge function $\xi(\rho)$ is hidden in the difference $\Delta(\rho) \equiv \xi_{1}-\xi(\rho)$ which appears both explicitly and as the argument of the space-dependent weak isospin matrix

$$
I_{G}(x)=\left(\begin{array}{cc}
-\sin (x) & -i \cos (x) \mathrm{e}^{i n \varphi} \\
i \cos (x) \mathrm{e}^{-i n \varphi} & \sin (x)
\end{array}\right)
$$


Imposing the boundary conditions $\xi(0)=0$ and $\xi(\infty)=\xi_{1}$ for the new gauge function $\xi(\rho)$ defines a well-behaved scattering problem. Otherwise, the specific form of $\xi(\rho)$ is irrelevant. This property allows us to verify our numerical results by modifying its shape while keeping the boundary conditions fixed.

All explicit matrices in eq. (19) act in spinor space. Together with the boundary conditions, eq. (3) a well-behaved scattering problem is obtained. With this choice of gauge a scattering matrix and, more generally, a Jost function can be straightforwardly computed. Moreover, the Born series to these scattering data can be constructed simply by iterating $H_{\text {int }}$.

Note that the gauge transformation is single-valued at spatial infinity, $U(\infty, \varphi)=U(\infty, \varphi+2 \pi)$. In this respect it differs from the analogous problem of fractional fluxes in QED. In that case a similar choice of gauge is hence not a remedy; rather the calculation of the vacuum polarization energy requires the introduction of a return flux to arrive at a well-behaved scattering problem 28 . The return-flux approach can also be used for the present calculation, but it is much more laborious numerically $19 \mid 20$.

\subsection{Fake Boson Field}

The idea of utilizing a fake boson field to simplify the treatment of higher order divergences was first implemented in Ref. 29. As mentioned above, the continuation to imaginary momenta $k \rightarrow i t$ and $\delta_{\ell}(k) \rightarrow \nu(t)$, where $\nu$ is the logarithm of the Jost function, allows the exchange of the momentum integral with the angular momentum sum. Then the third and fourth order contribution from the Born series produce logarithmic divergences. These divergences are similar to the ones found in the second order vacuum polarization energy of a boson field fluctuating about a scalar potential. Matching its strength appropriately allows to replace

$$
\sum_{\ell} D_{\ell}\left[\frac{d}{d t} \nu_{\ell}(t)\right]_{N} \rightarrow \frac{d}{d t}\left[\sum_{\ell} D_{\ell}\left(\nu_{\ell}(t)-\nu_{\ell}^{(1)}(t)-\nu_{\ell}^{(2)}(t)\right)-\sum_{\ell} \bar{D}_{\ell} \bar{\nu}_{\ell}^{(2)}(t)\right]
$$

under the integral in eq. (17). The quantity $E^{(N)}$ with this replacement will be called $E_{\delta}$. The over-bared quantities refer to the bosonic scattering data. The replacement eq. (21) must, of course, be accompanied by the boson Feynman diagram $E_{\mathrm{B}}$ so that the total vacuum polarization energy becomes

$$
E_{\mathrm{vac}}=E_{\delta}+E_{\mathrm{FD}}^{\mathrm{ren}}+E_{\mathrm{B}}^{\mathrm{ren}},
$$

where the superscript indicates the inclusion of the counterterm contributions. Each of the three terms on the right hand side of equation (22) is ultra-violet finite by itself. The advantage of eq. (22) and the replacement eq. (21) is now obvious: Instead of fermionic contributions up to order $N=4$, we only need to compute second order fermionic and bosonic Feynman diagrams and terms in the corresponding Born series. 


\section{Numerical Results for the Vacuum Polarization Energy}

Numerical results as well as dimensionfull parameters are measured in appropriate units of the (perturbative) fermion mass $m$.

We focus on the contribution of fermion fluctuations to the vacuum polarization energy because it dominates the boson counterpart by a factor proportional to the number of internal degrees of freedom, e.g. $N_{C}$, the number of colors. In this scenario we are now prepared to compute the vacuum polarization energy of a prescribed string configuration.

\subsection{Variational Ansätze}

Despite of the simplification in eq. (22), the numerical computation is still expensive. The scattering data are extracted from a multi-channel problem and for the final result to be reliable a huge number of partial wave must be included. This numerical effort restricts the number of variational parameters that can be used to characterize the profile functions. We have already introduced the strength parameter $\xi_{1}$. In addition, we introduce three scale parameters $w_{H}, w_{W}$ and $w_{\xi}$ via the ansätze

$$
f_{H}(\rho)=1-\mathrm{e}^{-\frac{\rho}{w_{H}}}, \quad f_{G}(\rho)=1-\mathrm{e}^{-\left(\frac{\rho}{w_{G}}\right)^{2}}, \quad \xi(\rho)=\xi_{1}\left[1-\mathrm{e}^{-\left(\frac{\rho}{w_{\xi}}\right)^{2}}\right] .
$$

The scale $w_{\xi}$ parameterizes the shape of the gauge profile. As explained above, this shape and thus $w_{\xi}$ should not be observable. The other specifics of the profiles are chosen to keep $E_{\mathrm{cl}}$ regular.

We have also considered an exponential parameterization for the gauge field

$$
f_{G}(\rho)=1-\left(1+\frac{\rho}{w_{G}}\right) \exp \left(-\frac{\rho}{w_{G}}\right)
$$

which yields a slightly better agreement with the original Nielsen-Olesen profiles that minimize $E_{\mathrm{cl}}$ for $\xi_{1}=\pi / 2$. No significant difference in $E_{\mathrm{vac}}$ was found between these ansätze.

\subsection{Gauge Invariance}

We check gauge invariance by varying the shape of the gauge profile, $\xi(\rho)$. A typical result is shown in table 1 . As expected, the individual contributions to $E_{\mathrm{vac}}$ depend

Table 1. Numerical results for the various contributions (22) to the fermion vacuum polarization energy in the minimal subtraction scheme.

\begin{tabular}{l|lll|l}
$w_{\xi}$ & $E_{\mathrm{FD}}^{\mathrm{ren}}$ & $E_{\delta}$ & $E_{\mathrm{B}}^{\mathrm{ren} .}$ & $E_{\mathrm{vac}}$ \\
\hline 0.5 & -0.2515 & 0.3489 & 0.0046 & 0.1020 \\
1.0 & -0.0655 & 0.1606 & 0.0032 & 0.0983 \\
2.0 & -0.0358 & 0.1294 & 0.0038 & 0.0974 \\
3.0 & -0.0320 & 0.1235 & 0.0056 & 0.0971 \\
4.0 & -0.0302 & 0.1193 & 0.0080 & 0.0971
\end{tabular}


strongly on $w_{\xi}$. However, these changes essentially compensate each other. Numerically the most cumbersome part of the calculation is $E_{\delta}$. From various numerical considerations (change of extrapolation scheme for partial wave sum, modification of momentum integration grid, etc.) its numerical accuracy is estimated to be at the $1 \%$ level. Within that range $E_{\mathrm{vac}}$ is independent of $w_{\xi}$, thus verifying gauge invariance.

The above results are obtained in the $\overline{\mathrm{MS}}$ renormalization scheme, which essentially omits the non-divergent parts of the Feynman diagrams. Any other scheme merely differs by manifestly gauge invariant (finite) counterterms.

\subsection{On-Shell Renormalization}

With the above mentioned choice of units, the dependence of $E_{\mathrm{vac}}$ on the model parameters factorizes in the $\overline{\mathrm{MS}}$ scheme which simplifies the computation because this dependence can easily be traced from $E_{\mathrm{cl}}$. However, for physically meaningful results we need to impose renormalization conditions that correspond to a particle interpretation, inducing a mild parameter dependence in $E_{\mathrm{CT}}$. To be specific we consider the so-called on-shell scheme in which the coefficients of the four allowed counterterms are determined such that

- the tadpole graph vanishes

- the Higgs mass remains unchanged

- the normalization of Higgs particle remains unchanged

- and the normalization of vector meson remains unchanged

in the presence of fermionic quantum corrections. Note that the vector meson mass $M_{W}$ is not fixed by these conditions and thus will be a prediction that includes quantum corrections. Hence we tune the gauge coupling to reproduce the physical value for $M_{W}$. Typical results for the vacuum polarization energy per unit length of the string are shown in figure 1, as functions of the variational parameters. Except for narrow string configurations dominated by the Higgs field, the vacuum polarization energy turns out to be positive. Therefore, fermionic quantum corrections do not provide any sensible binding and no stable uncharged string is found for the physically motivated parameters, eq. (7), for which $E_{\mathrm{cl}}$ dominates the total energy. Yet, $E_{\mathrm{cl}}$ deceases quickly with increasing Yukawa coupling $f$ and some stability is indeed seen for large $f$ and narrow strings. Unfortunately, in this regime the restriction to one fermion loop in the vacuum polarization energy is unreliable because of the occurrence of the Landau ghost 30 .

\section{Charged Strings}

Cosmic strings induce many fermionic bound state levels (whose energies are denoted by $\epsilon_{j}$ ) for the two-dimensional scattering problem. For $\xi_{1}=\pi / 2$ there even exists an exact zero mode ${ }^{12}$. In the three dimensional problem these bound states 
acquire a longitudinal momentum for the motion along the symmetry axis and their energies become

$$
E_{i}\left(p_{n}\right)=\sqrt{\epsilon_{i}^{2}+p_{n}^{2}} \quad \text { with } \quad p_{n}=\frac{n \pi}{L} .
$$

Here $L$ is the length of the string. In leading order of the $L \rightarrow \infty$ limit the sum over the discrete longitudinal momentum turns into a continuum integral, $\sum_{n} \longrightarrow \frac{L}{\pi} \int d p$. To minimize the bound state contribution a chemical potential $\mu$ with $\max \left(\left|\epsilon_{j}\right|\right) \leq \mu \leq m$ is introduced and all levels with $E_{i}(p) \leq \mu$ are populated. This procedure defines a Fermi momentum for each level, $p_{i}^{F}(\mu)=\sqrt{\mu^{2}-\epsilon_{i}^{2}}$ which enters the total charge per unit length of the string

$$
Q(\mu)=\sum_{i} \frac{p_{i}^{F}(\mu)}{\pi}
$$

This relation can be inverted to give $\mu=\mu(Q)$ and thus $p_{i}^{F}=p_{i}^{F}(Q)$. From this the binding energy (per unit length) for a prescribed charge

$$
E_{\text {bind }}(Q)=\frac{1}{\pi} \sum_{i} \int_{0}^{p_{i}^{F}(Q)} d p\left[\sqrt{\epsilon_{i}^{2}+p^{2}}-m\right]
$$

is computed relative to an equal number of free fermions that have energy $m$ each. Figure 2 shows the fermion contribution to the binding energy, $E_{\mathrm{vac}}+E_{\mathrm{bind}}(Q)$. For a given configuration the graph terminates at the point when all available bound state levels are occupied. For small charges narrow strings are favorable while the binding energy of strings with larger widths decreases more quickly as $Q$ increases. Surprisingly, the envelope along which $E_{\mathrm{vac}}+E_{\mathrm{bind}}$ is minimal forms a straight line. Extrapolating this line to $Q=0$ indicates that the fermion vacuum polarization energy should (approximately) vanish. This extrapolation circumvents the Landau ghost problem.

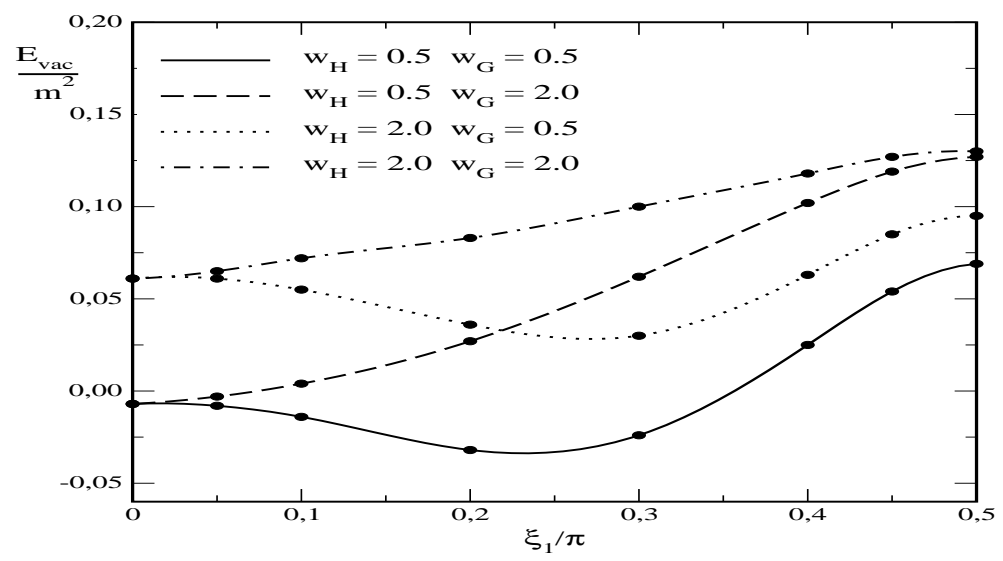

Fig. 1. Fermion vacuum polarization energy in the on-shell renormalization scheme. 
To finally decide on dynamical stability, the classical energy must be included. To this end we scan through several hundred configurations characterized by the variational ansätze, eq. (23). We label them by $s=1,2, \ldots$ and compute their total binding energy

$$
E_{\text {tot }}^{(s)}(Q)=E_{\mathrm{cl}}^{(s)}+N_{C}\left[E_{\mathrm{vac}}^{(s)}+E_{\text {bind }}^{(s)}(Q)\right]
$$

for a given charge. If

$$
E_{\mathrm{tot}}(Q):=\min _{s}\left[E_{\mathrm{tot}}^{(s)}(Q)\right]<0
$$

a stable configuration is constructed. Figure 3 shows $E_{\text {tot }}$ as a function of charge for various values of the Yukawa coupling constant, i.e. the mass of a non-interacting fermion. For $f \approx 1.6$ the classical and fermion energies essentially cancel each other and leave $E_{\text {tot }}$ roughly charge independent $c$. Bound objects are observed by further increasing the Yukawa coupling to about $f \approx 1.7$, which corresponds to a heavy fermion mass which is less than twice the top quark mass. We find that the minimizing configurations have $\xi_{1} \approx 0$, i.e. they are dominated by the Higgs field.

\section{Conclusion}

We have presented and discussed the formalism to compute the fermion contribution to the vacuum polarization energy per unit length of an infinitely long straight string in a simplified version of the electroweak standard model. Our approach is

${ }^{c}$ The exhibited dependence at small $Q$ is artificial because very narrow strings have not been considered to avoid the Landau ghost inconsistency.

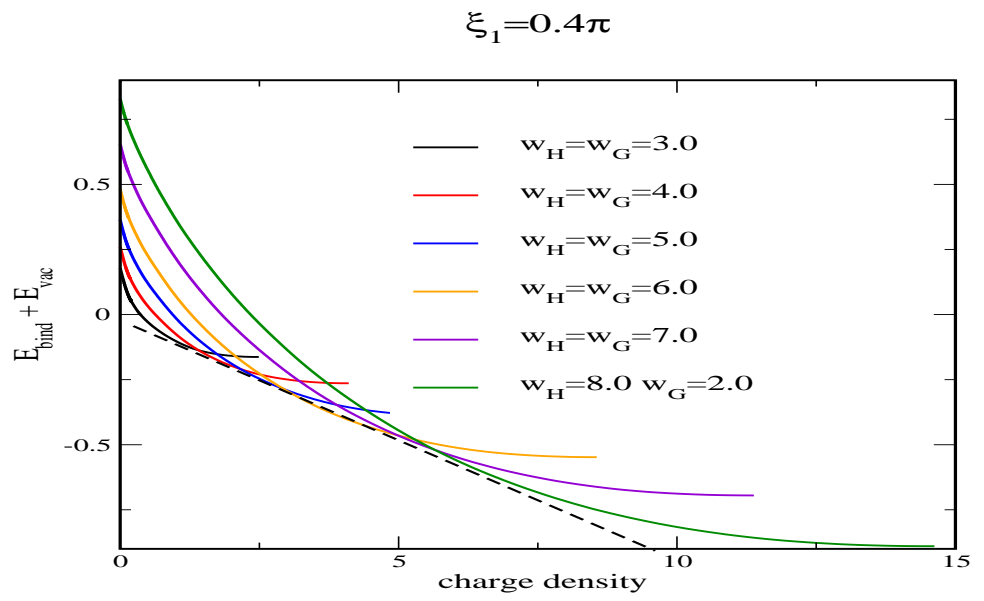

Fig. 2. (Color online) Fermion contribution to the energy of a charged string. 
based on the interface formalism, for which the analytical properties of scattering data are essential. We have also seen that a particular subset of gauge choices circumvents obstacles that in a naïve treatment arise from the non-trivial structure of the string configuration at spatial infinity. Numerically we have found that the vacuum polarization is small and positive in the regime in which the one-fermion loop approximation is reliable. Hence, there is no quantum stabilization of the string. However, we have seen that a heavy fermion doublet can stabilize a nontrivial string background for a non-zero fixed charge per unit length. The resulting configuration is dominated by the Higgs field. Since any additional variational degree of freedom can only lower the total energy, the embedding of this configuration in the full standard model, with the $U(1)$ gauge field included, will also yield a bound object as long as mixing between this heavy and the standard model fermions can be ignored. We see binding set in at $m \approx 300 \mathrm{GeV}$, which is still within the range of energy scales at which the standard model is expected to provide an effective description of the relevant physics, and also within the range to be probed at the LHC. Light fermions would contribute only weakly to the binding of the string, since their Yukawa couplings are small. As a result, we can add them to our model, e.g. to accommodate anomaly cancellation, without significantly changing the result.

\section{Acknowledgments}

One of us (HW) is grateful to the organizers for putting together this enjoyable conference and providing the opportunity to present this project.

This work is founded in parts by the NRF (South Africa). NG acknowledges support under NSF grant PHY08-55426.

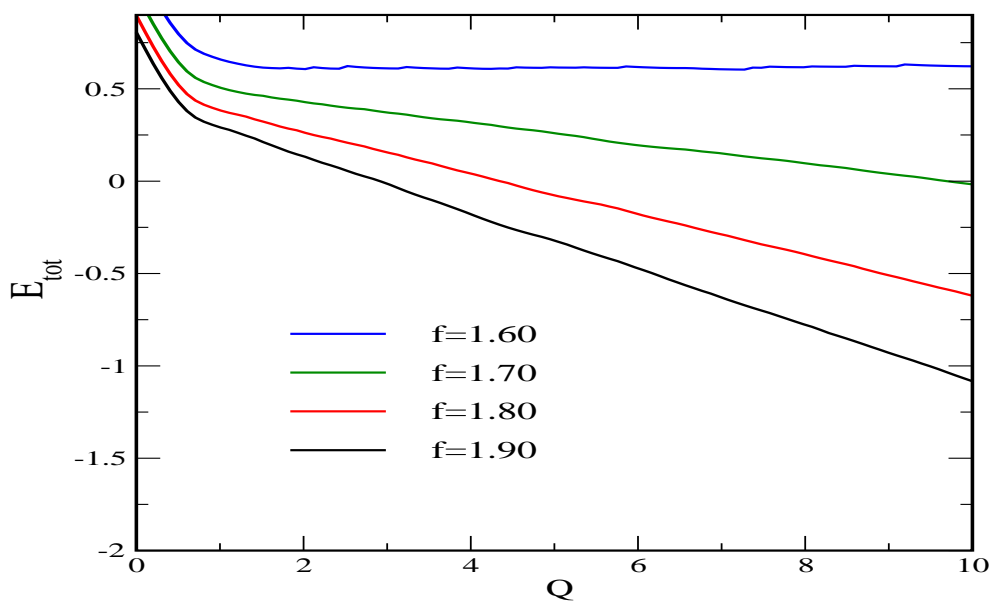

Fig. 3. (Color online) Total energy of the charged string. 


\section{References}

1. T. Vachaspati, Phys. Rev. Lett. 68, 1977 (1992) [Erratum-ibid. 69, 216 (1992)].

2. A. Achucarro and T. Vachaspati, Phys. Rept. 327, 347 (2000).

3. Y. Nambu, Nucl. Phys. B 130, 505 (1977).

4. H. B. Nielsen and P. Olesen, Nucl. Phys. B 61, 45 (1973).

5. A. Achucarro and C. J. A. Martins, arXiv:0811.1277 [astro-ph].

6. E. J. Copeland and T. W. B. Kibble, Proc. Roy. Soc. Lond. A 466, 623 (2010).

7. R.H. Brandenberger and A. Davis, Phys. Lett. B 308, 79 (1993);

8. R.H. Brandenberger, A. Davis, and M. Trodden, Phys. Lett. B 335, 123 (1994).

9. K. Kajantie, M. Laine, K. Rummukainen, and M. E. Shaposhnikov, Phys. Rev. Lett. 77 , 2887 (1996); K. Rummukainen, M. Tsypin, K. Kajantie, M. Laine, and M. E. Shaposhnikov, Nucl. Phys. B 532 , 283 (1998); F. Csikor, Z. Fodor, and J. Heitger, Phys. Rev. Lett. 82,21 (1999).

10. C. Grojean, G. Servant, and J. D. Wells, Phys. Rev. D 71, 036001 (2005); A. Menon, D. E. Morrissey, and C. E. M. Wagner, Phys. Rev. D 70, 035005 (2004).

11. E. D'Hoker and E. Farhi, Nucl. Phys. B 248, 59 (1984); 77 (1984).

12. S. G. Naculich, Phys. Rev. Lett. 75, 998 (1995).

13. F. R. Klinkhamer and C. Rupp, J. Math. Phys. 44, 3619 (2003).

14. G. Starkman, D. Stojkovic, and T. Vachaspati, Phys. Rev. D 65, 065003 (2002); G. Starkman, D. Stojkovic, and T. Vachaspati, Phys. Rev. D 63, 085011 (2001); D. Stojkovic, Int. J. Mod. Phys. A 16S1C, 1034 (2001).

15. M. Groves and W. B. Perkins, Nucl. Phys. B 573, 449 (2000).

16. M. Bordag and I. Drozdov, Phys. Rev. D 68, 065026 (2003).

17. J. Baacke and N. Kevlishvili, Phys. Rev. D 78, 085008 (2008).

18. M. Lilley, F. Di Marco, J. Martin, and P. Peter, Phys. Rev. D 82, 023510 (2010).

19. H. Weigel, M. Quandt, N. Graham, and O. Schröder, Nucl. Phys. B 831, 306 (2010).

20. H. Weigel and M. Quandt, Phys. Lett. B 690, 514 (2010).

21. H. Weigel, M. Quandt, and N. Graham, Phys. Rev. Lett. 106, 101601 (2011).

22. N. Graham, M. Quandt, and H. Weigel, Phys. Rev. D 84, 025017 (2011).

23. N. Graham, M. Quandt, and H. Weigel, Lect. Notes Phys. 777, 1 (2009).

24. N. Graham, R. L. Jaffe, M. Quandt, and H. Weigel, Phys. Rev. Lett. 87, 131601 (2001).

25. M. G. Krein, Mat. Sborn. (N.S.) 33597 (1953); M. G. Krein, Sov. Math.-Dokl. 3 707 (1962); M. Sh. Birman and M. G. Krein, Sov. Math.-Dokl. 3740 (1962).

26. N. Graham, R. L. Jaffe, V. Khemani, M. Quandt, M. Scandurra, and H. Weigel, Nucl. Phys. B 645, 49 (2002).

27. R. D. Puff, Phys. Rev. A 11, 154 (1975); N. Graham, R. L. Jaffe, M. Quandt, and H. Weigel, Annals Phys. 293, 240 (2001).

28. N. Graham, V. Khemani, M. Quandt, O. Schröder, and H. Weigel, Nucl. Phys. B 707, 233 (2005); H. Weigel, J. Phys. A 39, 6799 (2006).

29. E. Farhi, N. Graham, R. L. Jaffe, and H. Weigel, Nucl. Phys. B 630, 241 (2002).

30. G. Ripka and S. Kahana, Phys. Rev. D 36, 1233 (1987); J. Hartmann, F. Beck, and W. Bentz, Phys. Rev. C 50, 3088 (1994). 